\title{
Long non-coding RNA ADAMTS9-AS2 inhibits liver cancer cell proliferation, migration and invasion
}

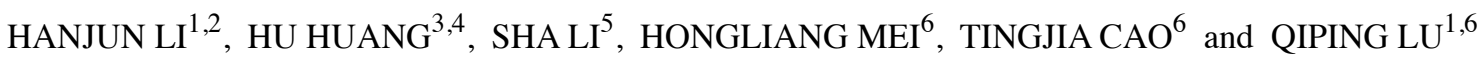 \\ ${ }^{1}$ The First School of Clinical Medicine, Southern Medical University, Guangzhou, Guangdong 510515; \\ ${ }^{2}$ Department of Pancreatic Surgery, Renmin Hospital of Wuhan University, Wuhan, Hubei 430060; \\ ${ }^{3}$ Cancer Center, Daping Hospital and Research Institute of Surgery, Third Military Medical University, \\ Chongqing 400042; ${ }^{4}$ Department of Oncology, The People's Liberation Army No. 161 Hospital, Wuhan, \\ Hubei 430010; Departments of ${ }^{5}$ Anesthesiology and ${ }^{6}$ General Surgery, General Hospital of Central \\ Theater Command of The People's Liberation Army, Wuhan, Hubei 430070, P.R. China
}

Received January 5, 2020; Accepted July 21, 2020

DOI: $10.3892 /$ etm.2021.9991

\begin{abstract}
Long non-coding RNA (lncRNA) ADAM metallopeptidase with thrombospondin type 1 motif 9 antisense RNA 2 (ADAMTS9-AS2) is involved in various types of cancer, such as ovarian cancer, lung cancer and clear cell renal cell carcinoma. However, the roles of ADAMTS9-AS2 in liver cancer are not completely understood. The present study aimed to determine the functional role of ADAMTS9-AS2 in human liver cancer and investigate the potential underlying molecular mechanisms. The expression levels of ADAMTS9-AS2 and ADAMTS9 were determined following ADAMTS9-AS2 overexpression and knockdown. The results indicated that ADAMTS9-AS2 overexpression and knockdown increased and decreased ADAMTS9 mRNA and protein expression levels, respectively, indicating that alterations in ADAMTS9 expression corresponded with ADAMTS9-AS2 expression. Subsequently, the effects of ADAMTS9-AS2 on liver cancer cell proliferation, migrationandinvasionwereanalyzedbyperformingCellCounting Kit-8, wound healing and Transwell assays, respectively. The results demonstrated that ADAMTS9-AS2 inhibited liver cancer cell proliferation, migration and invasion. Finally, the effect of ADAMTS9 on PI3K/AKT/mTOR signaling pathway-associated
\end{abstract}

Correspondence to: Dr Qiping Lu, The First School of Clinical Medicine, Southern Medical University, 1838 North Guangzhou Avenue, Guangzhou, Guangdong 510515, P.R. China

E-mail: ptwklqp111@163.com

Abbreviations: lncRNA, long non-coding RNA; ADAMTS9, ADAM metallopeptidase with thrombospondin type 1 motif 9; ADAMTS9-AS2, ADAMTS9 antisense RNA 2; siRNA, small interfering RNA; RT-qPCR, reverse transcription-quantitative PCR

Key words: IncRNA ADAMTS9-AS2, proliferation, $\mathrm{PI} 3 \mathrm{~K} / \mathrm{AKT} / \mathrm{mTOR}$, autophagy, liver cancer cells proteins [AKT, phosphorylated-AKT, phosphatidylinositol-4, 5-bisphosphate 3 -kinase catalytic subunit $\beta$ (PIK3CB), mTOR and phosphorylated-mTOR], several key autophagy-related proteins [light chain 3-I/II (LC3-I/II), beclin 1 (BECN1) and sequestosome 1 (SQSTM1)] and apoptosis-related proteins (Bax and Bcl-2) was detected via western blotting. The results suggested that ADAMTS9-AS2 downregulated the phosphorylation of AKT and mTOR, the protein expression level of PIK3CB, as well as the expression levels of autophagy protein SQSTM1 and antiapoptotic protein Bcl-2. By contrast, ADAMTS9-AS2 upregulated the expression levels of autophagy proteins LC3-II and BECN1, and the proapoptotic protein Bax. Collectively, ADAMTS9-AS2 inhibited liver cancer cell proliferation, migration and invasion via inhibiting the PI3K/AKT/mTOR signaling pathway. The present study provided a novel insight into the role of ADAMTS9-AS2 in liver cancer.

\section{Introduction}

Liver cancer is the second leading cause of cancer-associated death worldwide $(1,2)$. The burden of liver cancer is globally increasing and its survival rate is $<10 \%$ (3). In the past years, improvements in patient outcomes have been demonstrated for patients with early stage liver cancer (4); however, the development of treatment resistance is common (5), therefore, the development of novel therapeutic strategies is important.

Long non-coding RNAs (lncRNAs), a type of non-coding RNA (ncRNA), are defined as transcribed RNA molecules of $>200$ nucleotides in length (6). The roles of lncRNAs in cancer treatment have attracted increasing attention $(7,8)$. Research has suggested that lncRNAs have multiple biological effects on tumor development, cell differentiation and metabolism (9). For instance, Jiang et al (10) identified that lncRNA differentiation antagonizing ncRNA promoted osteosarcoma proliferation, migration and invasion in vitro. Another study conducted by $\mathrm{Zhu}$ and $\mathrm{Xu}$ (11) revealed that downregulation of lncRNA Angelman syndrome chromosome region promoted osteoblast differentiation. Furthermore, lncRNA-associated regulatory processes may have an inhibitory effect on liver 
cancer cells (12). In particular, a common type of cancerassociated lncRNA is an antisense partner of a proteincoding gene, for example, HOXD antisense growth-associated long non-coding RNA (13) and zinc finger E-box binding homeobox 1-AS1 (14).

lncRNA ADAM metallopeptidase with thrombospondin type 1 motif 9 antisense RNA 2 (ADAMTS9-AS2) is an antisense transcript of protein-coding gene ADAMTS9. The ADAMTS9 lncRNA/mRNA gene pair is located at chromosome 3p14.1, a region that is absent in hereditary renal cancer (15). In addition, several studies have indicated that ADAMTS9-AS2 is involved in gastric cancer development via activation of the PI3K/AKT signaling pathway (16), in lung cancer progression via inhibiting microRNA-223-3p and promoting transforming growth factor- $\beta$ receptor 3 (17), and in salivary adenoid cystic carcinoma metastasis via PI3K/AKT and mitogen-activated protein kinase kinase/ERK signaling (18). However, whether ADAMTS9-AS2 is associated with liver cancer is not completely understood.

In the present study, the association between ADAMTS9-AS2 and ADAMTS9 was investigated. The functional role of ADAMTS9-AS2 in liver cancer cells was explored in terms of its effect on cell proliferation, migration and invasion. Finally, the association between ADAMTS9-AS2 and the PI3K/AKT/mTOR signaling pathway, autophagy and apoptosis was studied in HepG2 and MHCC97-H cells. The present study furthered the current understanding of the mechanism underlying ADAMTS9-AS2 in liver cancer and, therefore, may provide a potential biomarker and therapeutic target for liver cancer.

\section{Materials and methods}

Cell culture and treatment. A normal liver cell line (MIHA) and liver cancer cell lines (HepG2, MHCC97-H and Hep 3B2.1-7) were obtained from The Cell Bank of Type Culture Collection of the Chinese Academy of Sciences. STR profiling was performed to authenticate the HepG2 cell line. Cells were cultured in DMEM (Gibco; Thermo Fisher Scientific, Inc.) supplemented with 10\% FBS (Gibco; Thermo Fisher Scientific, Inc.) and $100 \mathrm{U} / \mathrm{ml}$ penicillin/streptomycin at $37^{\circ} \mathrm{C}$ with $5 \%$ $\mathrm{CO}_{2}$. Cells were plated in 24 -well plates $\left(2.5 \times 10^{5}\right.$ cells $/$ well $)$.

Reverse transcription-quantitative PCR (RT-qPCR). The expression levels of ADAMTS9-AS2 and ADAMTS9 were detected via RT-qPCR as described previously (19). Briefly, total RNA was extracted from cells using TRIzol ${ }^{\text {ब }}$ reagent (Thermo Fisher Scientific, Inc.) according to the manufacturer's instructions. Subsequently, total RNA (1 $\mu \mathrm{g})$ was reverse transcribed into cDNA using a PrimeScript RT Reagent kit with cDNA Eraser (Takara Biotechnology Co. Ltd.) at $37^{\circ} \mathrm{C}$. Subsequently, qPCR was performed using SYBR Premix Ex Taq (Takara Biotechnology Co., Ltd.) on an ABI 7900 system (Applied Biosystems; Thermo Fisher Scientific, Inc.). The following thermocycling conditions were used for qPCR: Pre-denaturation at $95^{\circ} \mathrm{C}$ for $10 \mathrm{~min}$; followed by 40 cycles at $95^{\circ} \mathrm{C}$ for $5 \mathrm{sec}, 58^{\circ} \mathrm{C}$ for $10 \mathrm{sec}$, and $72^{\circ} \mathrm{C}$ for $25 \mathrm{sec} ; 95^{\circ} \mathrm{C}$ for $30 \mathrm{sec}, 58^{\circ} \mathrm{C}$ for $5 \mathrm{sec}$ and $95^{\circ} \mathrm{C}$ for $30 \mathrm{sec}$. The following primers were used for qPCR: ADAMTS9-AS2 forward, 5'-AAGAAACCCTGATGTCTGGCTGAA-3' and reverse, 5'-GTGTTACTTGAGGAGAAAGCGAAA-3'; ADAMTS9 forward, 5'-TGGGTTTTCCAGTTTTCAG-3' and reverse, 5'-GTTGATGCTAAAACGACCC-3'; and GAPDH forward, 5'-ACGGATTTGGTCGTATTGGGCG-3' and reverse, 5'-GCTCCTGGAAGATGGTGATGGG-3'. mRNA expression levels were quantified using the $2^{-\Delta \Delta \mathrm{Cq}} \mathrm{Ct}$ method (20) and normalized to the internal reference gene GAPDH.

Plasmid construction and cell transfection. pcDNA3.1-ADAMTS9-AS2 (pcDNA3.1-AS2), a plasmid containing ADAMTS9-AS2, was constructed by cloning the fragment of ADAMTS9-AS2 into a pcDNA3.1 vector (Invitrogen; Thermo Fisher Scientific, Inc.) at the BamHI-EcoRI sites. Small interfering (si)RNAs targeting ADAMTS9-AS2 (si-1-AS2 and si-2-AS2) and the siRNA negative control (NC; si-NC) were designed and synthesized by Guangzhou RiboBio Co., Ltd. Cells were seeded ( $5 \times 10^{5}$ cells/well) into 12 -well plates and cultured for at least $24 \mathrm{~h}$ prior to transfection. Cells were transfected with pcDNA3.1-AS2, pcDNA3.1 (300 ng), si-AS2-1, si-AS2-2 or si-NC (40 pmol) using Lipofectamine ${ }^{\circledR} 2000$ (Invitrogen; Thermo Fisher Scientific, Inc.) according to the manufacturer's protocol. At $48 \mathrm{~h}$ post-transfection, cells were used for subsequent experiments. The target sequences of the vectors and siRNAs were as follows: pcDNA3.1-AS2 forward, 5'-GGGGTACCAAACTTGACGTACACACG-3' and reverse,AGCCGGAATTCTTTTCTGTTTTTATAA TGTAC-3'; si-AS2-1, 5'-GCATGACGCAACTTTGCTA-3'; si-AS2-2, 5'-CCTGTCTACAGGCTGATAT-3'; si-NC: 5'-TTC TCCGAACGTGTCACGTTT-3'.

Cell Counting Kit-8 (CCK-8) assay. To evaluate cell proliferation, the CCK-8 assay (Dojindo Molecular Technologies, Inc.) was performed according to the manufacturer's protocol. In brief, cells were seeded $\left(3 \times 10^{3}\right.$ cells/well) into 96 -well plates and cultured for $18 \mathrm{~h}$ prior to transfection. Cells were transfected with pcDNA3.1-AS2, pcDNA3.1 (100 ng), si-AS2-2 or si-NC (5 pmol) using Lipofectamine RNAiMAX (0.3 $\mu \mathrm{l} / \mathrm{well})$. At 4-6 $\mathrm{h}$ post-transfection, the culture medium was replaced with DMEM containing 10\% CCK-8 solution and incubated at $37^{\circ} \mathrm{C}$ for $1 \mathrm{~h}$. At $0-3$ days post-transfection, the optical density value was measured at a wavelength of $450 \mathrm{~nm}$ using a Multiskan FC (Thermo Fisher Scientific, Inc.).

Wound healing assay. Cell migration was assessed by performing a wound healing assay. Briefly, prior to transfection (pcDNA 3.1-AS2, pcDNA 3.1, si-AS2 or si-NC), cells were plated into 6-well plates and scraped with a pipette tip to generate uniform wounds in each well after cell culture for $18 \mathrm{~h}$. Cells were cultured in medium supplemented with $2 \%$ FBS. At $0,24,48$ and $72 \mathrm{~h}$, the wounds were observed using an inverted microscope (magnification, $\mathrm{x} 40$ ). Image $\mathrm{J}$ (National Institutes of Health; v1.8.0.112) was used for statistical analysis.

Transwell assay. Cell invasion was determined by performing a Transwell assay using Transwell inserts (Corning, Inc.) with a Matrigel-precoated membrane filter (pore width, $8 \mu \mathrm{m}$ ) for $2 \mathrm{~h}$ at $37^{\circ} \mathrm{C}$. At $24 \mathrm{~h}$ post-transfection, cells density of $4 \times 10^{5}$ in $100 \mu \mathrm{l}$ serum-free DMEM were plated in the upper chambers. DMEM supplemented with 10\% FBS was plated in the lower chambers. Following incubation for $48 \mathrm{~h}$, cells on 
the upper surface of the membrane were removed using cotton swabs. Invading cells were fixed in $4 \%$ polyformaldehyde for $10 \mathrm{~min}$ and stained with $0.1 \%$ crystal violet for $20 \mathrm{~min}$. at room temperature. Invading cells were counted in five randomly selected fields of view (magnification, $\mathrm{x} 40$ ) using an inverted microscope.

Western blotting. The protein expression levels of ADAMTS9, AKT, phosphorylated (p)-AKT phosphatidylinositol-4,5-bisphosphate 3-kinase catalytic subunit $\beta$ (PIK3CB), mTOR, p-mTOR, light chain (LC)3-I/II, beclin 1 (BECN1), sequestosome 1 (SQSTM1), Bax and Bcl-2 were detected via western blotting. Transfected cells were washed with pre-cooled PBS on ice and boiled in SDS-sample buffer (Western and IP cell lysate, Sangon Biotech, Co., Ltd; cat. no. C500035). Proteins (30 $\mu \mathrm{g}$ per lane) were separated via 10\% SDS-PAGE and transferred to PVDF membranes. After blocking with $5 \%$ skimmed milk for $90 \mathrm{~min}$ at room temperature, the membranes were incubated overnight at $4^{\circ} \mathrm{C}$ with primary antibodies (all 1:1,000; ABclonal Biotech Co., Ltd.) targeted against: ADAMTS9 (cat. no. A17928), AKT (cat. no. A11016), p-AKT (cat. no. AP0140), PIK3CB (cat. no. A11906), mTOR (cat. no. A11355), p-mTOR (cat. no. AP0 409), LC3-I/II (cat. no. A11282), BECN1 (cat. no. A10101), SQSTM1 (cat. no. A11483), Bax (cat. no. A12009), Bcl-2 (cat. no. A11025) and GAPDH (cat. no. AC002). Subsequently, the membranes were incubated at $37^{\circ} \mathrm{C}$ for $1 \mathrm{~h}$ with horseradish peroxidaselabeled Goat Anti-Mouse IgG (cat. no. AS003; 1:10,000; ABclonal Biotech Co., Ltd.) or Goat Anti-Rabbit IgG (cat. no. AS014; 1:10,000; ABclonal Biotech Co., Ltd.) secondary antibody. Protein bands were visualized using ECL chemiluminescent reagent (EMD Millipore) and the Bio-Rad Gel Doc $\mathrm{XR}^{+}$system (Bio-Rad Laboratories, Inc.). The BCA method was used to determine protein concentration, and GAPDH was used as the loading control. ImageJ (National Institutes of Health; v1.8.0.112) was used for analysis.

Statistical analysis. Statistical analyses were conducted using GraphPad Prism software (version 7.0; GraphPad Software, Inc.). Data are expressed as the mean \pm standard deviation. Each experiment was repeated three times. Comparisons between two groups were analyzed using an unpaired Student's t-test. Comparisons among multiple groups were analyzed using one-way ANOVA followed by Dunnett's post hoc test or two-way ANOVA followed by Sidak's post hoc test. $\mathrm{P}<0.05$ was considered to indicate a statistically significant difference.

\section{Results}

ADAMTS9-AS2 is positively associated with ADAMTS9 expression in liver cancer cell lines. To investigate the association between the expression of ADAMTS9-AS2 and ADAMTS9 in liver cancer cell lines, the expression of ADAMTS9-AS2 was detected in a normal liver cell line (MIHA) and three liver cancer cell lines (HepG2, MHCC97-H and Hep 3B2.1-7) via RT-qPCR. Compared with MIHA cells, ADAMTS9-AS2 expression levels were significantly upregulated in Hep 3B2.1-7 cells $(\mathrm{P}<0.001)$ and

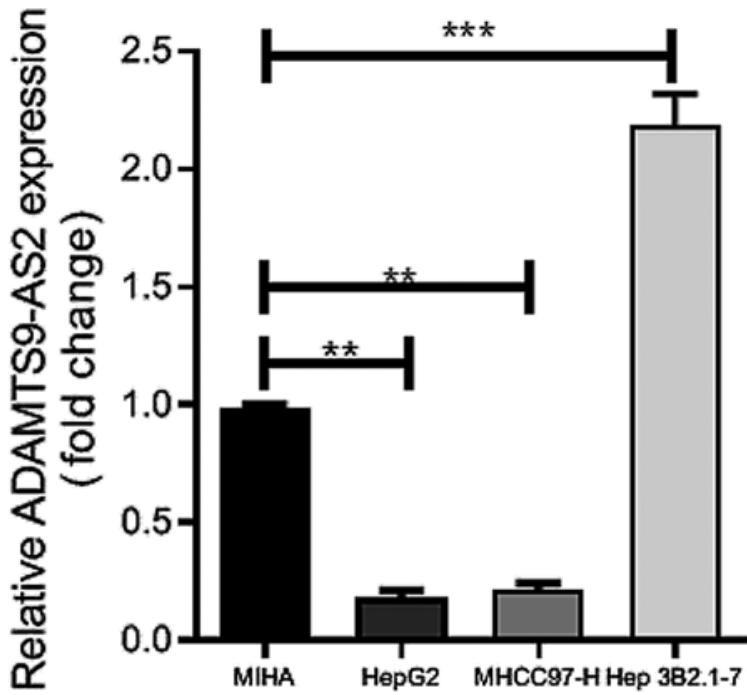

Figure 1. Expression of ADAMTS9-AS2 in a normal liver cell line and three liver cancer cell lines. ${ }^{* *} \mathrm{P}<0.01$ and ${ }^{* * *} \mathrm{P}<0.001$ vs. MIHA. ADAMTS9-AS2, ADAM metallopeptidase with thrombospondin type 1 motif 9 antisense RNA 2.

significantly downregulated in HepG2 and MHCC97-H cells (both $\mathrm{P}<0.01$ ) (Fig. 1). Therefore, HepG2 and MHCC97-H cell lines were selected for subsequent experiments. ADAMTS9 expression levels were measured following ADAMTS9-AS2 overexpression and knockdown in HepG2 and MHCC97-H cells. pcDNA3.1-AS2, si-1-AS2 and si-2-AS2 were constructed and transfected into HepG2 and MHCC97-H cells. The results indicated that ADAMTS9-AS2 expression was significantly increased in the pcDNA3.1-AS2 group compared with the pcDNA3.1 group (HepG2 and MHCC97-H, P<0.001; Fig. S1). By contrast, ADAMTS9-AS2 expression was significantly decreased in the si-AS2-1 and si-AS2-2 groups compared with the si-NC group (HepG2, $\mathrm{P}<0.001$; MHCC97-H, $\mathrm{P}<0.01$; Fig. S1). As the transfection efficiency of si-AS2-2 was higher compared with si-AS2-1, si-AS2-2 was selected and labeled as si-AS2 for subsequent experiments.

Furthermore,ADAMTS9mRNA and protein expression levels were significantly higher in the pcDNA3.1-AS2 group compared with the pcDNA3.1 group $(\mathrm{P}<0.01$; Fig. $2 \mathrm{~A}-\mathrm{D})$, suggesting that ADAMTS9-AS2 overexpression upregulated ADAMTS9 expression. Conversely, ADAMTS9 mRNA and protein expression levels were significantly decreased in the si-AS2 group compared with the si-NC group (all $\mathrm{P}<0.05$; Fig. 2A-D), indicating that ADAMTS9-AS2 knockdown downregulated ADAMTS9 expression. The results indicated that ADAMTS9-AS2 overexpression or knockdown resulted in increased and decreased ADAMTS9 expression levels, respectively.

ADAMTS9-AS2 inhibits liver cancer cell proliferation, migration and invasion. To investigate the functional roles of ADAMTS9-AS2 in liver cancer, ADAMTS9-AS2 overexpression and knockdown were performed in HepG2 and MHCC97-H cell lines to determine the effect on cell proliferation, migration and invasion. The CCK-8 assay suggested that pcDNA3.1-AS2 significantly inhibited HepG2 cell proliferation compared with the pcDNA3.1 group ( 24 and $48 \mathrm{~h}, \mathrm{P}<0.05$; 72 h, P<0.01; Fig. 3A). By contrast, HepG2 cell proliferation 
A

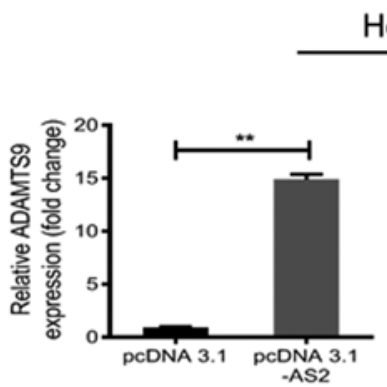

C

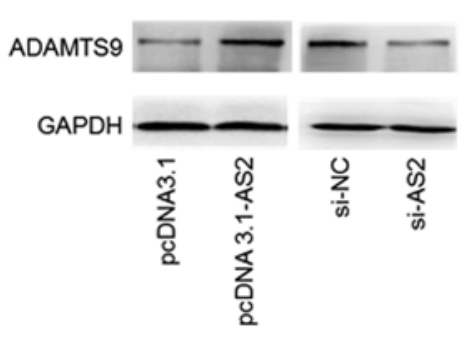

HepG2
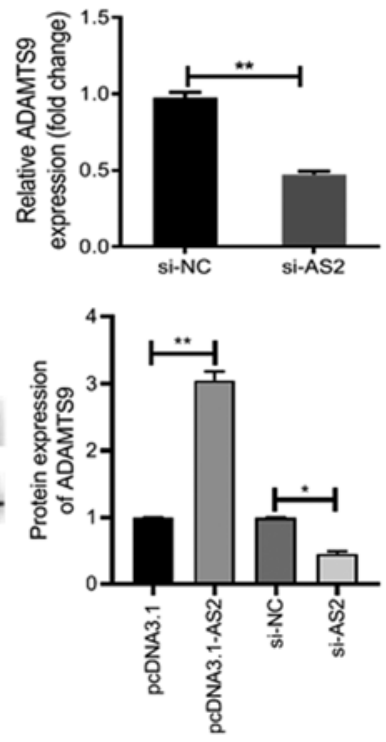

B

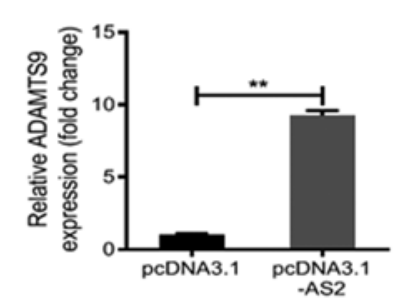

D

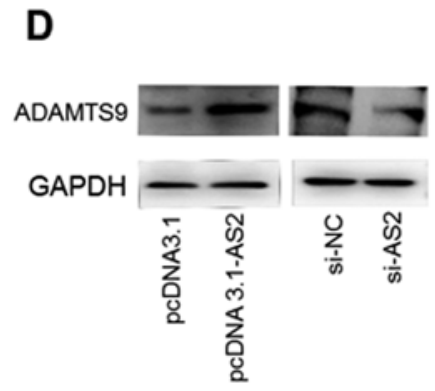

MHCC97-H
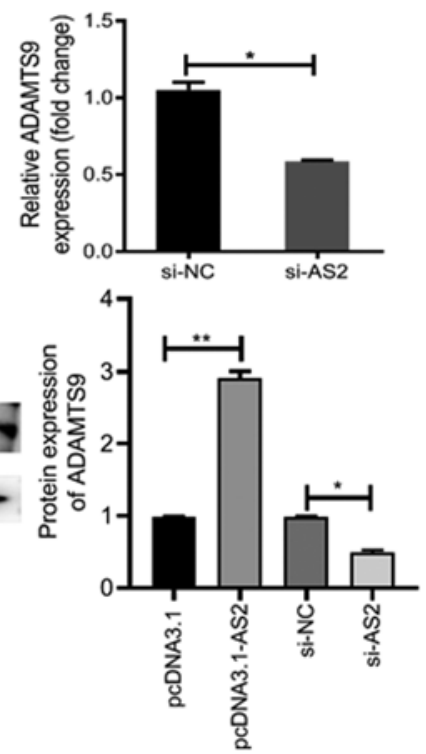

Figure 2. Association between the expression of ADAMTS9 and ADAMTS9-AS2. Transfection efficiency of pcDNA 3.1-AS2 and si-AS2 in (A) HepG2 and (B) MHCC97-H cells. ADAMTS9 protein expression levels in (C) HepG2 and (D) MHCC97-H cells following transfection. " P $<0.05$ and ${ }^{* *} \mathrm{P}<0.01$. ADAMTS9, ADAM metallopeptidase with thrombospondin type 1 motif 9; AS2, antisense RNA 2; si, small interfering RNA; NC, negative control.

was significantly increased in the si-AS2 group compared with the si-NC group ( 48 and $72 \mathrm{~h}, \mathrm{P}<0.05$; Fig. 3A). Similar results were obtained in the MHCC97-H cell line ( $\mathrm{P}<0.01$; Fig. 3B).

In addition, the results of the wound healing assay suggested that HepG2 cell migration was significantly decreased in the pcDNA3.1-AS2 group compared with the pcDNA3.1 group $(\mathrm{P}<0.01)$, but significantly increased in the si-AS2 group compared with the si-NC group at 24, 48 and $72 \mathrm{~h}(\mathrm{P}<0.05$; Fig. 3C). The results obtained for the MHCC97-H cell line were consistent with the results obtained in HepG2 cells (P<0.05; Fig. 3D).

Furthermore, the results of the Transwell assay indicated that HepG2 cell invasion was significantly increased in the pcDNA3.1-AS2 group compared with the pcDNA3.1 group $(\mathrm{P}<0.01$; Fig. 3E). Conversely, HepG2 cell invasion was significantly enhanced in the si-AS2 group compared with the si-NC group $(\mathrm{P}<0.01 ;$ Fig. 3E). Similar results were obtained in the MHCC 97-H cell line ( $<<0.01$; Fig. 3F). The results of the aforementioned assays indicated that ADAMTS9-AS2 inhibited liver cancer cell proliferation, migration and invasion.

ADAMTS9-AS2 inhibits the PI3K/AKT/mTOR signaling pathway in liver cancer cells. As an important signaling pathway, the PI3K/AKT signaling pathway is involved in cancer metastasis and invasion $(21,22)$. To explore whether an association existed between ADAMTS9-AS2 and the PI3K/AKT signaling pathway, the expression levels of key molecules involved in the signaling pathway were detected in HepG2 and MHCC97-H cells via western blotting after ADAMTS9-AS2 knockdown or overexpression. No marked differences in the protein expression levels of AKT and mTOR were observed between the pcDNA3.1-AS2 and the pcDNA3.1 group. Similar results were observed in the si-AS2 compared with the si-NC group (Fig. 4A). The expression levels of
p-AKT/AKT, PIK3CB and p-mTOR/mTOR were significantly reduced in the pcDNA3.1-AS2 group compared with the pcDNA3.1 group $(\mathrm{P}<0.05, \mathrm{P}<0.01$ and $\mathrm{P}<0.01$, respectively), whereas the expression levels were significantly increased in the si-AS2 group compared with the si-NC group (all $\mathrm{P}<0.05$; Fig. 4A). The results obtained with the MHCC97-H cell line were consistent with the results obtained in HepG2 cells (all $\mathrm{P}<0.05$; Fig. 4B). Therefore, the results indicated that ADAMTS9-AS2 inhibited the PI3K/AKT/mTOR signaling pathway in liver cancer cells. ADAMTS9-AS2 is involved in autophagy and apoptosis in liver cancer cells, the $\mathrm{PI} 3 \mathrm{~K} / \mathrm{AKT} / \mathrm{mTOR}$ signaling pathway is closely associated with autophagy and the kinase mTOR is a major regulator of the autophagy process (23). Previous research has demonstrated that IncRNA pituary tumor-transforming 3 , pseudogene was involved in the cell cycle and apoptosis of liver cancer cells via the PI3K/AKT signaling pathway (24). Therefore, the present study investigated the association between ADAMTS9-AS2, autophagy and apoptosis in the present study. The expression levels of several key autophagy-related (LC3-I/II, BECN1 and SQSTM1) and apoptotic-related (Bax and $\mathrm{Bcl}-2)$ proteins in HepG2 and MHCC97-H cells were determined via western blotting. The results indicated that the expression levels of LC3-II and BECN1 were significantly increased, whereas SQSTM1 expression levels were significantly decreased in the pcDNA3.1-AS2 group compared with the pcDNA3.1 group $(\mathrm{P}<0.05, \mathrm{P}<0.01$ and $\mathrm{P}<0.05$, respectively; Fig. 5A). The opposite result was obtained in the si-AS2 group compared with the si-NC group (all $\mathrm{P}<0.05$; Fig. 5A). In the MHCC $97-\mathrm{H}$ cell line, the results were similar to the results obtained for HepG2 cells (all $\mathrm{P}<0.05$; Fig. 5B).

In addition, the expression of the proapoptotic protein Bax was significantly increased, whereas the expression of the antiapoptotic protein $\mathrm{Bcl}-2$ was significantly decreased 
HepG2

A

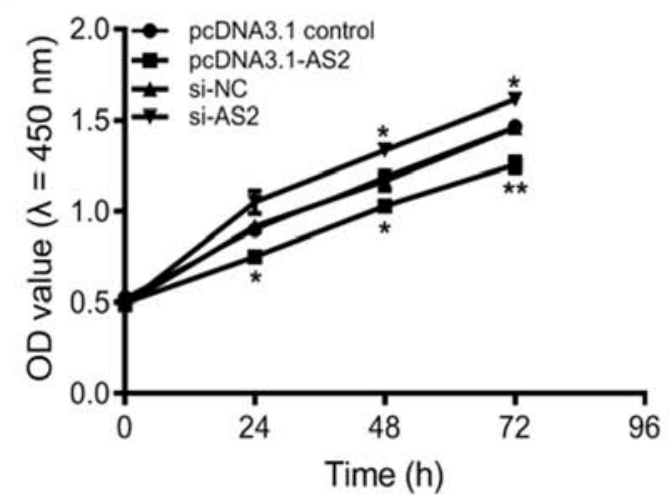

C
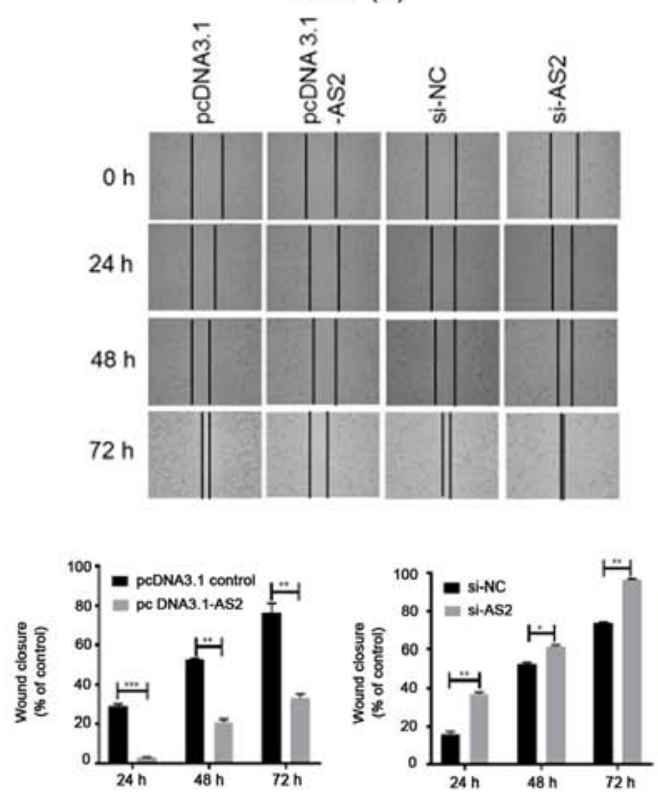

E

pcDNA3.1 pcDNA3.1-AS2

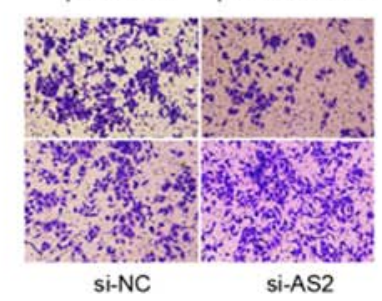

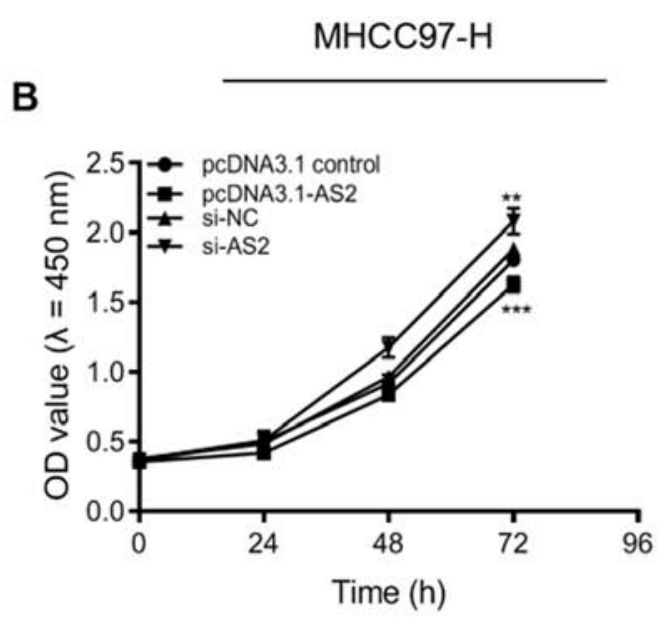

D
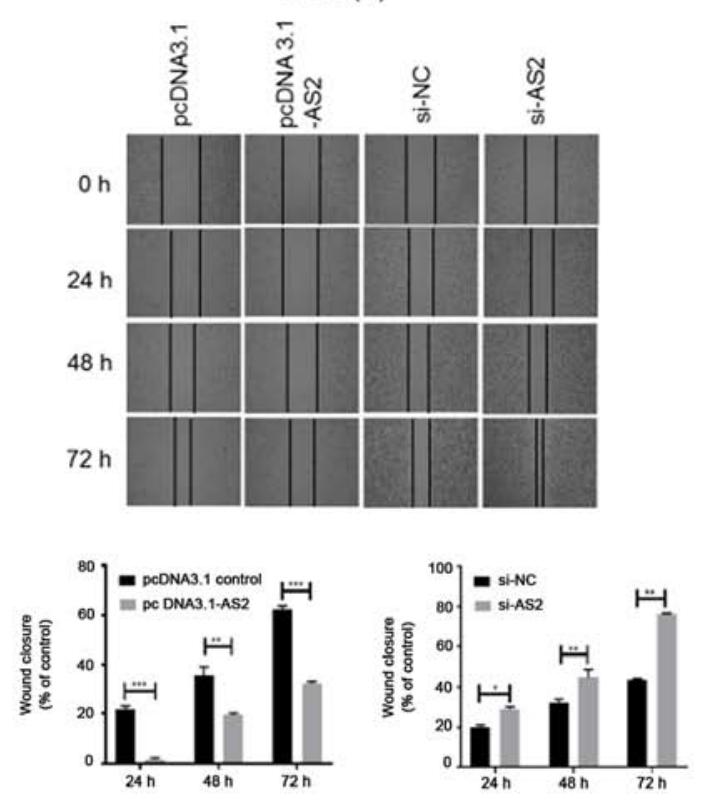

$\mathbf{F}$

pcDNA3.1 pcDNA3.1-AS2

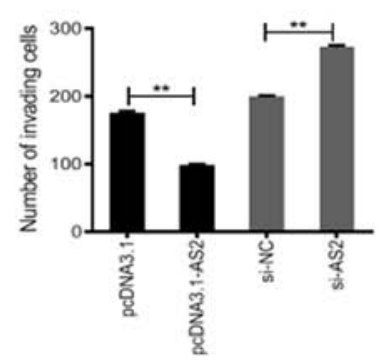

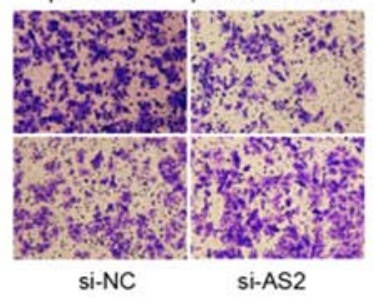

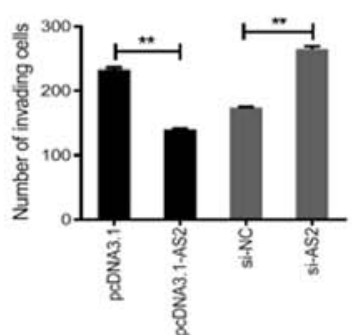

Figure 3. ADAMTS9-AS2 inhibits liver cancer cell proliferation, migration and invasion. (A) HepG2 and (B) MHCC97-H cell proliferation was assessed using a Cell Counting Kit-8 assay. (C) HepG2 and (D) MHCC97-H cell migration was assessed by performing a wound healing assay (magnification, $\mathrm{x} 40$ ). (E) HepG2 and (F) MHCC97-H cell invasion was analyzed using a Transwell assay (magnification, $\mathrm{x} 40$ ). ${ }^{*} \mathrm{P}<0.05,{ }^{* * *} \mathrm{P}<0.01$ and ${ }^{* * * *} \mathrm{P}<0.001$. ADAMTS9, ADAM metallopeptidase with thrombospondin type 1 motif 9; AS2, antisense RNA 2; OD, optical density; si, small interfering RNA; NC, negative control.

in the pcDNA3.1-AS2 group compared with the pcDNA3.1 group in HepG2 cells (both $\mathrm{P}<0.05$ ). The opposite effect was observed in the si-AS2 group compared with the si-NC group (both $\mathrm{P}<0.05$; Fig. 5C). Similar results were obtained for the MHCC97-H cell line (all $\mathrm{P}<0.05$; Fig. 5D). Collectively, the results indicated that ADAMTS9-AS2 upregulated ADAMTS9 expression, which inhibited the PI3K/AKT signaling pathway to promote autophagy, thereby inducing apoptosis, and inhibiting tumor cell migration and invasion.

\section{Discussion}

As reported by multiple studies, numerous lncRNAs have crucial roles in the pathogenesis of liver cancer $(25,26)$. ADAMTS9-AS2 is an antisense transcript of its adjacent protein-coding gene ADAMTS9. Numerous studies have reported that IncRNA ADAMTS9-AS2 is involved in various diseases, including gastric (16) and lung cancer (17). However, whether ADAMTS9-AS2 has a role in human liver cancer 
A
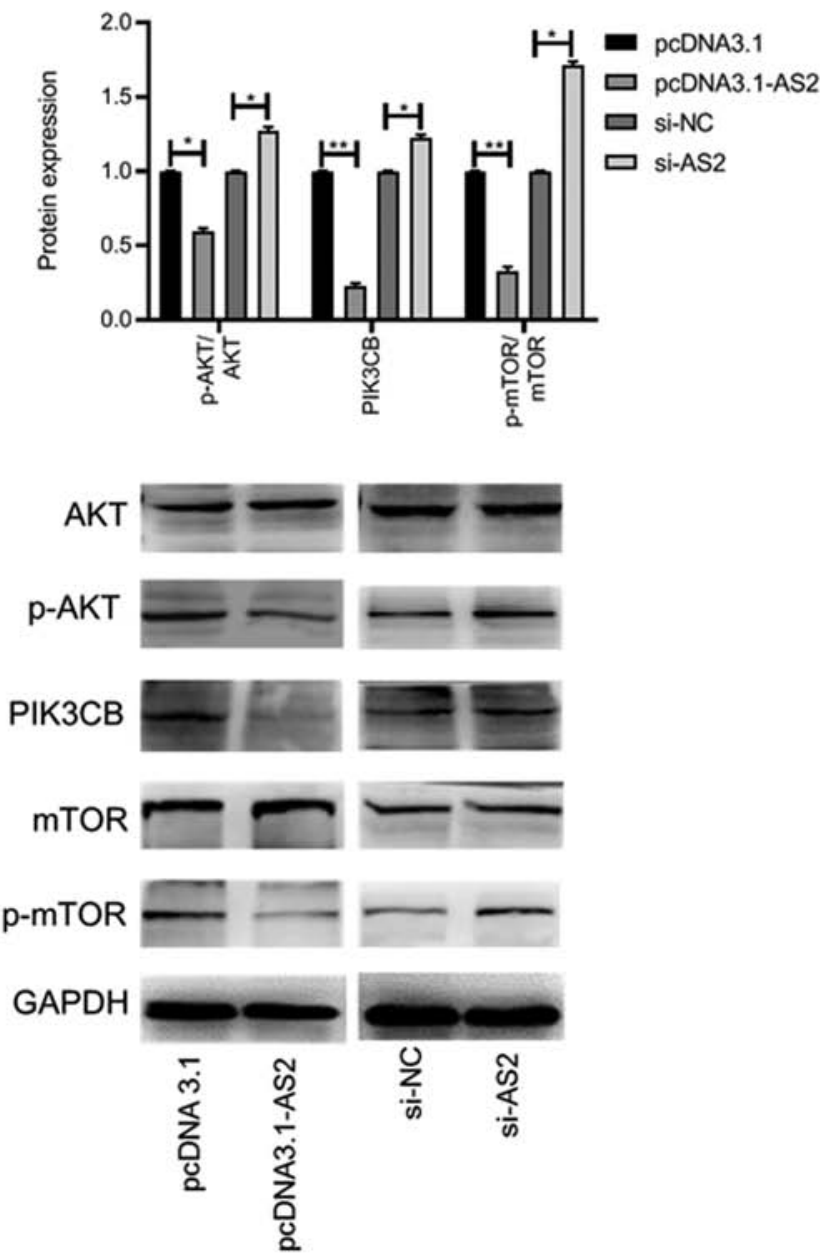

B
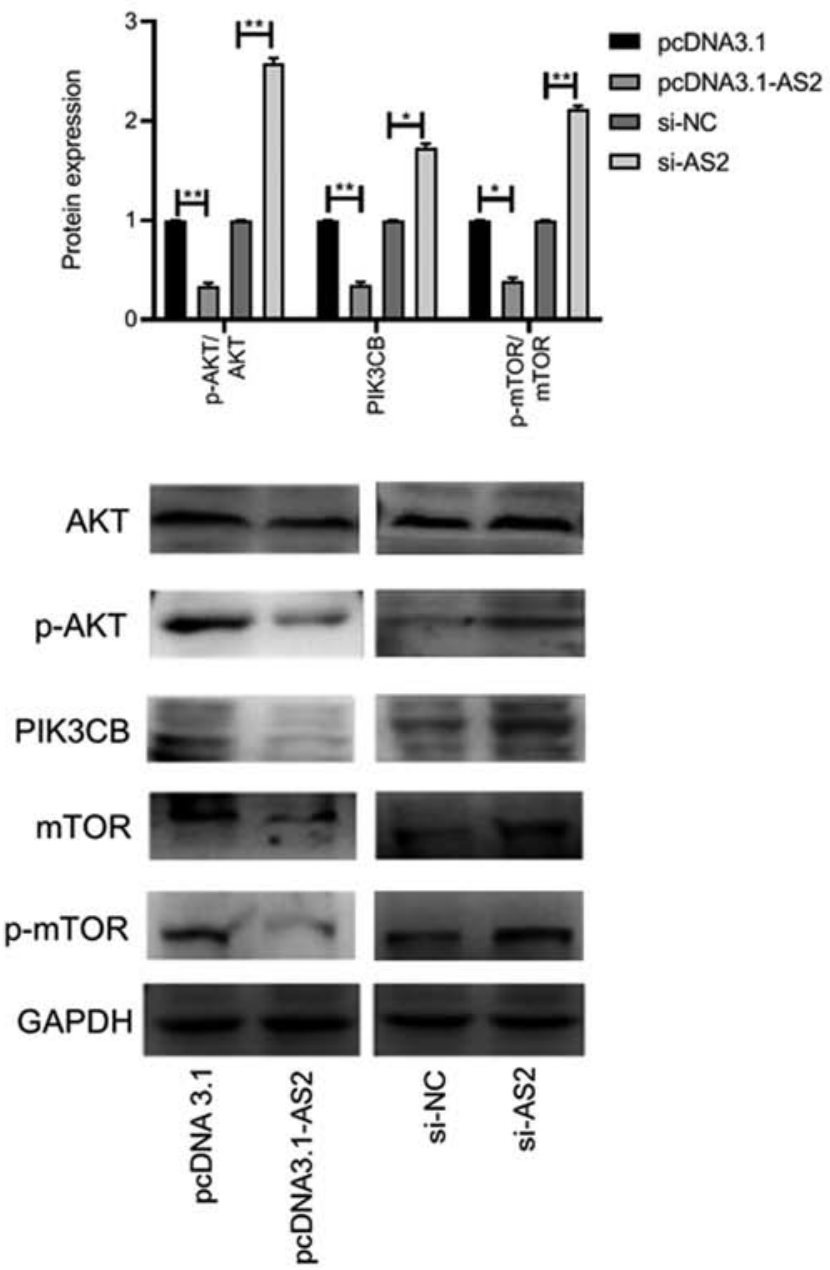

Figure 4. ADAMTS9-AS2 is associated with the PI3K/AKT/mTOR signaling pathway in liver cancer cells. Expression levels of AKT, p-AKT, PIK3CB, mTOR and p-mTOR in (A) HepG2 and (B) MHCC97-H cells were determined via western blotting. ${ }^{*} \mathrm{P}<0.05$ and ${ }^{* *} \mathrm{P}<0.01$. ADAMTS9, ADAM metallopeptidase with thrombospondin type 1 motif 9; AS2, antisense RNA 2; p, phosphorylated; PIK3CB, phosphatidylinositol-4,5-bisphosphate 3-kinase catalytic subunit $\beta$; si, small interfering RNA; NC, negative control.

is not completely understood. A previous study indicated that alterations in ADAMTS9 expression levels were consistent with alterations in ADAMTS9-AS2 expression levels in glioma (27). In the present study, a similar pattern was observed in liver cancer cells. In addition, ADAMTS9 was previously reported to act as a functional tumor suppressor by inhibiting the oncogenic AKT/mTOR signaling pathway (28). Therefore, it was hypothesized that ADAMTS9-AS2 may serve a role in the suppression of liver cancer. In a preliminary experiment, the present study assessed the expression of ADAMTS9-AS2 in three liver cancer cell lines and one normal hepatic cell line. The HepG2 and MHCC97-H cell lines were selected for subsequent experiments, as ADAMTS9-AS2 expression was significantly downregulated in the two cell lines compared with MIHA cells. It was further indicated that alterations in ADAMTS9 expression corresponded with ADAMTS9-AS2 expression levels in HepG2 and MHCC97-H cell lines. Subsequently, functional assays indicated that ADAMTS9-AS2 suppressed HepG2 and MHCC97-H cell proliferation, migration and invasion.
Further functional experiments indicated that ADAMTS9-AS2 decreased the expression of p-AKT, PIK3CB and p-mTOR, suggesting that ADAMTS9-AS2 was involved in the progression of liver cancer by regulating the PI3K/AKT/mTOR signaling pathway. The results of several previous studies support that the PI3K/AKT/mTOR signaling pathway is commonly associated with autophagy in liver cancer (29-32). Moreover, autophagy is important for maintaining the energy balance and stability of the cellular environment (33). In view of the crucial role of autophagy in the development and progression of various tumor types, targeting autophagy has been considered as a novel strategy for anticancer therapy $(34,35)$. In the present study, the effect of ADAMTS9-AS2 expression on several key autophagy-related (LC3-I/II, BECN1 and SQSTM1) $(36,37)$ and apoptosis-related (Bax and $\mathrm{Bcl}-2$ ) (38) proteins was explored. The results indicated that ADAMTS9-AS2 increased the expression of LC3-II and BECN1, but inhibited the expression of SQSTM1 in liver cancer cells. In terms of apoptotic proteins, ADAMTS9-AS2 increased 

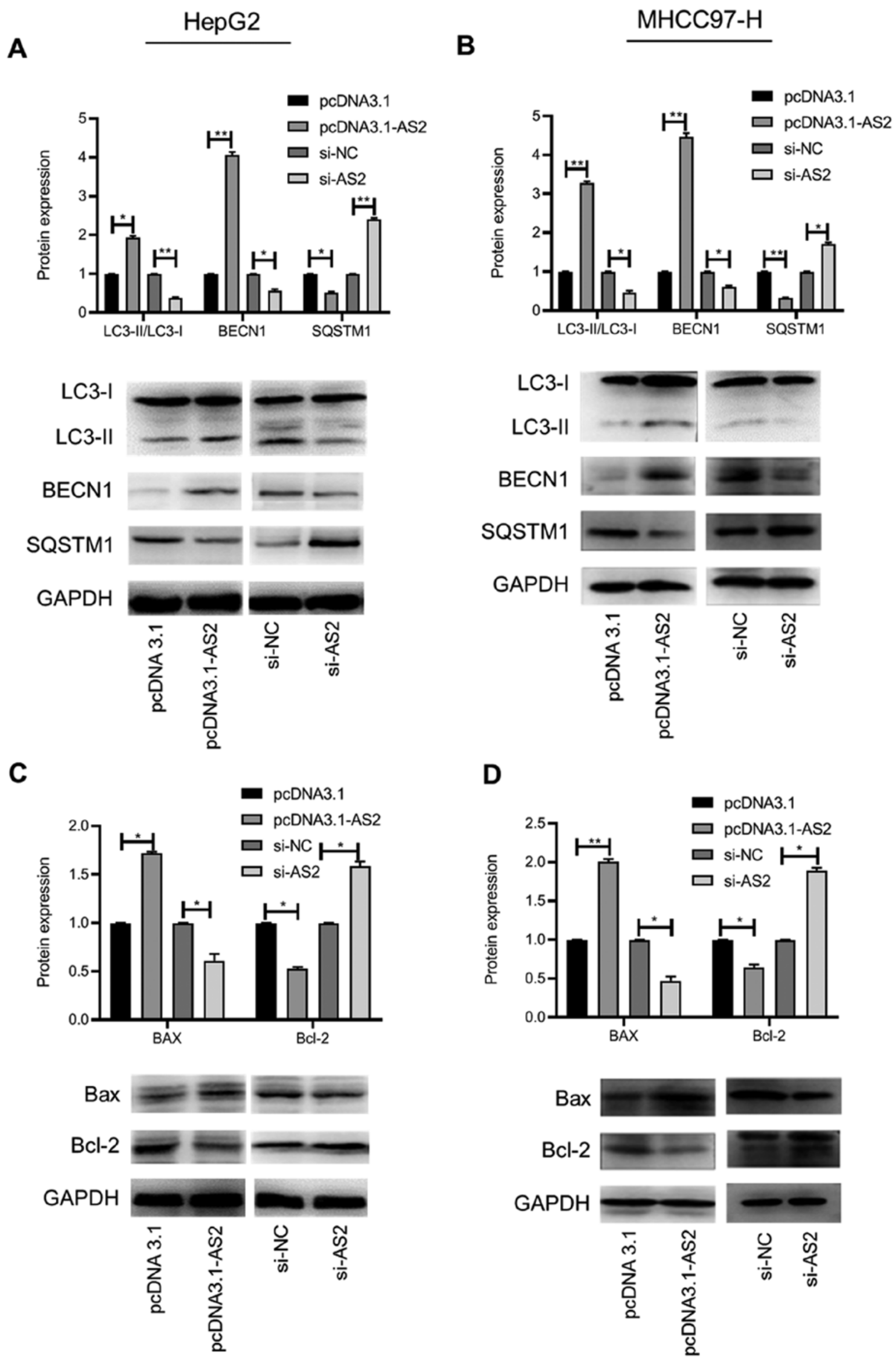

Figure 5. ADAMTS9-AS2 participates in liver cancer cell autophagy and apoptosis. Expression levels of autophagy-related proteins in (A) HepG2 and (B) MHCC97-H cells were determined via western blotting. Expression levels of apoptosis-related in (C) HepG2 and (D) MHCC97-H cells were determined via western blotting. " $\mathrm{P}<0.05$ and ${ }^{* *} \mathrm{P}<0.01$. ADAMTS9, ADAM metallopeptidase with thrombospondin type 1 motif 9; AS2, antisense RNA 2; si, small interfering RNA; NC, negative control; LC, light chain; BECN1, beclin 1; SQSTM1, sequestosome 1. 
the expression of pro-apoptotic Bax and decreased the expression of anti-apoptotic Bcl-2. The results suggested that ADAMTS9-AS2 inhibited the PI3K/AKT signaling pathway and promoted autophagy, thereby resulting in liver cancer cell apoptosis.

The results of the present study are limited as the experiments were only conducted in two cell lines. In addition, the mechanisms underlying ADAMTS9-AS2-mediated regulation of the PI3K/AKT/mTOR signaling pathway in liver cancer require further investigation. Furthermore, whether the effects of ADAMTS9 on various cell properties were due to its effects on the AKT/mTOR signaling pathway also requires further investigation.

In conclusion, the present study indicated that lncRNA ADAMTS9-AS2 might serve an inhibitory role in liver cancer cell lines. The results provided insight into the possible mechanism underlying the involvement of ADAMTS9-AS2 in the suppression of liver cancer, namely via cell autophagy and apoptosis as a result of regulating the PI3K/AKT/mTOR signaling pathway.

\section{Acknowledgements}

Not applicable.

\section{Funding}

Not applicable.

\section{Availability of data and materials}

The datasets used and/or analyzed during the current study are available from the corresponding author on reasonable request.

\section{Authors' contributions}

HL performed cell function and mechanistic assays. $\mathrm{HH}$ performed RT-qPCR. SL and HM performed the cell culturing and transfection experiments. TC conceived and designed the study, and coordinated and drafted the manuscript. QL supervised the study and contributed to analyzing the data. All authors read and approved the final manuscript.

\section{Ethics approval and consent to participate}

Not applicable.

\section{Patient consent for publication}

Not applicable.

\section{Competing interests}

The authors declare that they have no competing interests.

\section{References}

1. Torre LA, Bray F, Siegel RL, Ferlay J, Lortet-Tieulent J and Jemal A: Global cancer statistics, 2012. CA Cancer J Clin 65: 87-108, 2015.
2. Llovet JM, Zucman-Rossi J, Pikarsky E, Sangro B, Schwartz M, Sherman M and Gores G: Hepatocellular carcinoma. Nat Revi Dis Primers 2: 16018, 2016.

3. Wang $\mathrm{H}, \mathrm{Lu} \mathrm{Z}$ and Zhao X: Tumorigenesis, diagnosis, and therapeutic potential of exosomes in liver cancer. J Hematol Oncol 12: 133, 2019.

4. Llovet JM, Montal R, Sia D and Finn RS: Molecular therapies and precision medicine for hepatocellular carcinoma. Nat Rev Clin Oncol 15: 599-616, 2018.

5. Ma S, Sun J, Guo Y, Zhang P, Liu Y, Zheng D and Shi J: Combination of AAV-TRAIL with miR-221-Zip therapeutic strategy overcomes the resistance to TRAIL induced apoptosis in liver cancer. Theranostics 7: 3228-3242, 2017.

6. Chen Y, He Y and Zhou H: The potential role of lncRNAs in diabetes and diabetic microvascular complications. Endocr J 67: 659-668, 2020.

7. Wang KC and Chang HY: Molecular mechanisms of long noncoding RNAs. Mol Cell 43: 904-914, 2011.

8. Prensner JR and Chinnaiyan AM: The emergence of lncRNAs in cancer biology. Cancer Discov 1: 391-407, 2011.

9. Liz J and Esteller M: IncRNAs and microRNAs with a role in cancer development. Biochim Biophys Acta 1859: 169-176, 2016.

10. Jiang N, Wang X, Xie X, Liao Y, Liu N, Liu J, Miao N, Shen $\mathrm{J}$ and Peng T: lncRNA DANCR promotes tumor progression and cancer stemness features in osteosarcoma by upregulating AXL via miR-33a-5p inhibition. Cancer Lett 405: 46-55, 2017.

11. Zhu L and Xu PC: Downregulated LncRNA-ANCR promotes osteoblast differentiation by targeting EZH2 and regulating Runx2 expression. Biochem Biophys Res Commun 432: 612-617, 2013.

12. Li C, Chen J, Zhang K, Feng B, Wang $\mathrm{R}$ and Chen L: Progress and prospects of long noncoding RNAs (lncRNAs) in hepatocellular carcinoma. Cell Physiol Biochem 36: 423-434, 2015.

13. Wang H, Huo X, Yang XR, He J, Cheng L, Wang N, Deng X, Jin $\mathrm{H}$, Wang $\mathrm{N}$, Wang C, et al: STAT3-mediated upregulation of IncRNA HOXD-AS1 as a ceRNA facilitates liver cancer metastasis by regulating SOX4. Mol Cancer 16: 136, 2017.

14. Li T, Xie J, Shen C, Cheng D, Shi Y, Wu Z, Deng X, Chen $\mathrm{H}$, Shen B, Peng C, et al: Upregulation of long noncoding RNA ZEB1-AS1 promotes tumor metastasis and predicts poor prognosis in hepatocellular carcinoma. Oncogene 35: 1575-1584, 2016.

15. Clark ME, Kelner GS, Turbeville LA, Boyer A, Arden KC and Maki RA: ADAMTS9, a novel member of the ADAM-TS/metallospondin gene family. Genomics 67: 343-350, 2000.

16. Wang X, Luo G, Zhang K, Cao J, Huang C, Jiang T, Liu B, $\mathrm{Su} \mathrm{L}$ and Qiu Z: Hypoxic tumor-derived exosomal miR-301a mediates M2 macrophage polarization via PI3K $\gamma$ to promote pancreatic cancer metastasis. Cancer Res 78: 4586-4598, 2018.

17. Liu C, Yang Z, Deng Z, Zhou Y, Gong Q, Zhao R and Chen T: Upregulated IncRNA ADAMTS9-AS2 suppresses progression of lung cancer through inhibition of miR-223-3p and promotion of TGFBR3. IUBMB Life 70: 536-546, 2018.

18. Xie S, Yu X, Li Y, Ma H, Fan S, Chen W, Pan G, Wang W, Zhang $\mathrm{H}$, Li $\mathrm{J}$ and Lin Z: Upregulation of lncRNA ADAMTS9-AS2 promotes salivary adenoid cystic carcinoma metastasis via PI3K/Akt and MEK/Erk signaling. Mol Ther 26: 2766-2778, 2018.

19. Jin Z, Yao J, Xie N, Cai L, Qi S, Zhang Z and Li B: Melittin constrains the expression of identified key genes associated with bladder cancer. J Immunol Res 2018: 5038172, 2018.

20. Livak KJ and Schmittgen TD: Analysis of relative gene expression data using real-time quantitative PCR and the 2(-Delta Delta C(T)) method. Methods 25: 402-408, 2001.

21. Xiong J, Li Z, Zhang Y, Li D, Zhang G, Luo X, Jie Z, Liu Y, Cao Y, Le Z, et al: PRL-3 promotes the peritoneal metastasis of gastric cancer through the PI3K/Akt signaling pathway by regulating PTEN. Oncol Rep 36: 1819-1828, 2016.

22. Liu X, Zhang W, Guo H, Yue J and Zhuo S: miR-98 functions as a tumor suppressor in salivary adenoid cystic carcinomas. Onco Targets Ther 9: 1777-1786, 2016.

23. Shu Ting WFS: PI3K/Akt/mTOR signaling pathway and its role in autophagy and tumor. Chin J Biochem Mol Biol 32: 1192-1196, 2016. 
24. Huang JL, Cao SW, Ou QS, Yang B, Zheng SH, Tang J, Chen J, Hu YW, Zheng L and Wang Q: The long non-coding RNA PTTG3P promotes cell growth and metastasis via up-regulating PTTG1 and activating PI3K/AKT signaling in hepatocellular carcinoma. Mol Cancer 17: 93, 2018.

25. Qiu L, Tang Q, Li G and Chen K: Long non-coding RNAs as biomarkers and therapeutic targets: Recent insights into hepatocellular carcinoma. Life Sci 191: 273-282, 2017.

26. Hu W, Feng H, Xu X, Huang X, Huang X, Chen W, Hao L and Xia W: Long noncoding RNA FOXD2-AS1 aggravates hepatocellular carcinoma tumorigenesis by regulating the miR-206/MAP3K1 axis. Cancer Med 9: 5620-5631, 2020.

27. Yao J, Zhou B, Zhang J, Geng P, Liu K, Zhu Y and Zhu W: A new tumor suppressor LncRNA ADAMTS9-AS2 is regulated by DNMT1 and inhibits migration of glioma cells. Tumour Biol 35: 7935-7944, 2014.

28. Du W, Wang S, Zhou Q, Li X, Chu J, Chang Z, Tao Q, $\mathrm{Ng}$ EK, Fang J, Sung JJ and Yu J: ADAMTS9 is a functional tumor suppressor through inhibiting AKT/mTOR pathway and associated with poor survival in gastric cancer. Oncogene 32: 3319-3328, 2013

29. Li TT, Zhu D, Mou T, Guo Z, Pu JL, Chen QS, Wei XF and $\mathrm{Wu} \mathrm{ZJ}$ : IL-37 induces autophagy in hepatocellular carcinoma cells by inhibiting the PI3K/AKT/mTOR pathway. Mol Immunol 87: 132-140, 2017.

30. Wang P, Guo QS, Wang ZW and Qian HX: HBx induces HepG-2 cells autophagy through PI3K/Akt-mTOR pathway. Mol Cell Biochem 372: 161-168, 2013.

31. Wang S, Zhu M, Wang Q, Hou Y, Li L, Weng H, Zhao Y, Chen D, Ding H, Guo J and Li M: Alpha-fetoprotein inhibits autophagy to promote malignant behaviour in hepatocellular carcinoma cells by activating PI3K/AKT/mTOR signalling. Cell Death Dis 9: 1027, 2018.
32. Wang SS, Chen YH, Chen N, Wang LJ, Chen DX, Weng HL, Dooley $\mathrm{S}$ and Ding HG: Hydrogen sulfide promotes autophagy of hepatocellular carcinoma cells through the PI3K/Akt/mTOR signaling pathway. Cell Death Dis 8: e2688, 2017.

33. Singh R and Cuervo AM: Autophagy in the cellular energetic balance. Cell Metab 13: 495-504, 2011.

34. Hua F, Shang S and Hu ZW: Seeking new anti-cancer agents from autophagy-regulating natural products. J Asian Nat Prod Res 19: 305-313, 2017.

35. Li YY, Feun LG, Thongkum A, Tu CH, Chen SM, Wangpaichitr M, Wu C, Kuo MT and Savaraj N: Autophagic mechanism in anti-cancer immunity: Its pros and cons for cancer therapy. Int J Mol Sci 18: 1297, 2017.

36. Lamark T, Svenning S and Johansen T: Regulation of selective autophagy: The p62/SQSTM1 paradigm. Essays Biochem 61: 609-624, 2017.

37. He R, Peng J, Yuan P, Xu F and Wei W: Divergent roles of BECN1 in LC3 lipidation and autophagosomal function. Autophagy 11: 740-747, 2015.

38. Hassan M, Watari H, AbuAlmaaty A, Ohba Y and Sakuragi N: Apoptosis and molecular targeting therapy in cancer. Biomed Res Int 2014: 150845, 2014.

This work is licensed under a Creative Commons Attribution-NonCommercial-NoDerivatives 4.0 International (CC BY-NC-ND 4.0) License. 